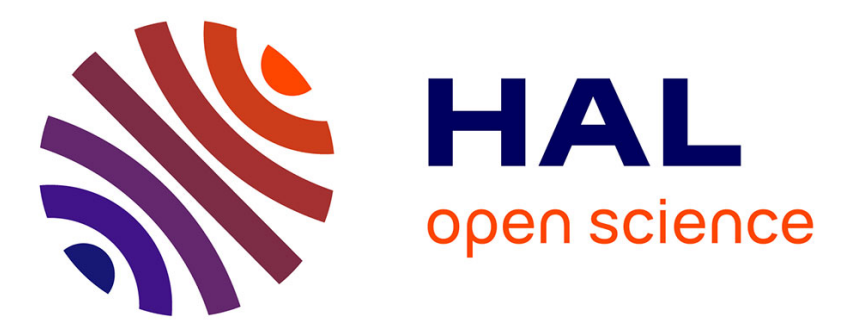

\title{
Model of surgical procedures for multimodal image-guided neurosurgery.
}

Pierre Jannin, Mélanie Raimbault, Xavier Morandi, Laurent Riffaud, Bernard

Gibaud

\section{- To cite this version:}

Pierre Jannin, Mélanie Raimbault, Xavier Morandi, Laurent Riffaud, Bernard Gibaud. Model of surgical procedures for multimodal image-guided neurosurgery.. Computer Aided Surgery, 2003, 8 (2), pp.98-106. inserm-00330538

\section{HAL Id: inserm-00330538 https://www.hal.inserm.fr/inserm-00330538}

Submitted on 14 Oct 2008

HAL is a multi-disciplinary open access archive for the deposit and dissemination of scientific research documents, whether they are published or not. The documents may come from teaching and research institutions in France or abroad, or from public or private research centers.
L'archive ouverte pluridisciplinaire HAL, est destinée au dépôt et à la diffusion de documents scientifiques de niveau recherche, publiés ou non, émanant des établissements d'enseignement et de recherche français ou étrangers, des laboratoires publics ou privés. 
Jannin P, Raimbault M, Morandi X, Riffaud L and Gibaud B. Models of Surgical Procedures for Multimodal Image-Guided Neurosurgery, Journal of Computer Aided Surgery, 2003;8(2):98-106

Models of Surgical Procedures for Multimodal Image-Guided Neurosurgery

\author{
P. Jannin ", Ph. D., M. Raimbault, M.Sc., X. Morandi, M.D., M.Sc., L. Riffaud, M.D., M.Sc., and B. \\ Gibaud Ph. D. \\ Laboratoire IDM, UPRES E.A. 3192, Université de Rennes (P.J.,M.R.,X.M.,L.R.,B.G.) and \\ Neurosurgery Department, University Hospital Rennes France (X.M.,L.R.)
}

\title{
Abstract
}

Objective: Improvement of the planning stage in image-guided surgery requires a better anticipation of the surgical procedure and its anatomical and functional environment. This anticipation should be provided by acquisition of multimodal medical images of the patient and by a better understanding of surgical procedures. In this paper, we propose improvements to the planning and performance of multimodal image-guided neurosurgery through the use of information models related to neurosurgical procedures.

Materials and Methods: We introduce a new generic model of surgical procedures in the context of multimodal image-guided craniotomies. The basic principle of the model is to break down the surgical procedure into a sequence of steps defining the surgical script. In the model, a step is defined by an action. The model assigns to each surgical step a list of image entities extracted from multimodal preoperative images (i.e. anatomical and/or functional images), which are relevant to the performance of that particular step. A semantic validation of the model was performed by instantiating the model entities for 29 surgical procedures.

Address correspondence/reprint requests to : Pierre JANNIN, Laboratoire IDM, UPRES E.A. 3192, Faculté de Médecine, Université de Rennes I, 2, Avenue du Pr. Léon Bernard CS34317 35043 Rennes cedex France ; EMail: pierre.jannin@univ-rennes1.fr; http://idm.univ-rennes1.fr A preliminary version of this paper was presented at the Fourth International Conference on Medical Image Computing and Computer-Assisted Intervention (MICCAI 2001), Utrecht, The Netherlands, October 2001 
Jannin P, Raimbault M, Morandi X, Riffaud L and Gibaud B. Models of Surgical Procedures for Multimodal Image-Guided Neurosurgery, Journal of Computer Aided Surgery, 2003;8(2):98-106

Results: The resulting generic model is described by a UML class diagram and a textual description. The validation has shown the relevance of the model, confirming the main assumptions underlying the model. It also provided us with some leads to improve the model.

Conclusion: While further validation is needed, the initial benefits of this approach can already be outlined. It should confer real added value to the different levels of image-guided surgery, from preprocessing to planning, as well as during surgery. Models of surgical procedures can manage image data according to the surgical script, which should lead to better anticipation of surgery through the development of simulation tools. Furthermore, they may improve the performance of surgery under microscope-based neuronavigation systems by making it possible to adapt both visualization and interaction features of multimodal preoperative images according to the model.

Keywords: Models of Surgical Procedures, UML, Multimodal Neuronavigation, Planning, Simulation, Image-Guided Neurosurgery

\section{Introduction}

Computer aided surgery has an increasingly role in surgery today. Computer aided surgery systems mostly require an initial planning stage based on multimodal imaging, the value of which has already been reported ${ }^{2,8,13,14}$. Expected progress with respect to planning include the improvement of the use and management of multimodal data, the planning of the whole surgical procedure (and not only a subset of it) and the involvement of technical, hardware and haptic constraints of the surgical procedure. As part of planning, patient data based simulation should allow surgical training for a given surgical performance. Most of the research on surgical simulation ${ }^{3,14}$ is more focused on realistic rendering, user interfaces or simulation of bio-mechanical reality than on understanding surgical procedures (i.e. identifying the core features of a surgical procedure). However, the need for models of the surgical procedure derived from task analysis was outlined for designing surgical simulation 
Jannin P, Raimbault M, Morandi X, Riffaud L and Gibaud B. Models of Surgical Procedures for Multimodal Image-Guided Neurosurgery, Journal of Computer Aided Surgery, 2003;8(2):98-106

systems ${ }^{24,25}$. Some authors recently reported the added value of models of surgical procedures for robotic systems ${ }^{12,19,20}$ or minimally invasive surgical procedures ${ }^{16}$. Neuronavigation for neurosurgery deals with complex multimodal information (i.e. anatomical and functional preoperative images of the patient $)^{1,17,21}$ and therefore models of surgical procedures may improve the management of this multimodal information especially with microscope-based augmented reality systems ${ }^{9}$.

In this paper, we propose a generic model for neurosurgical procedures in which the procedure is broken down into its main steps characterized by an action and related attributes. We studied three types of procedures, known to benefit from multimodal neuronavigation ${ }^{10,11}$, and accounting for approximately $75 \%$ of supratentorial procedures in our neurosurgery department (excluding traumatology, shunts, and vascular procedures): surgery for supratentorial intra-parenchymatous tumors: SSIT (67\%), surgery for supratentorial cavernomas: SSC (5\%) and selective amygdalo-hippocampectomies for medically intractable epilepsies: SAH (3\%). We also present the results of a semantic validation of the model performed on 29 clinical cases.

\section{Methods}

The basic principle of the model is to break down the surgical procedure into a sequence of major steps defining the surgical script. The model assigns to each surgical step a list of image entities extracted from multimodal preoperative images (i.e. anatomical and/or functional images), which are relevant to the performance of that particular step. The role of each image entity in a particular step is specified from a list of predefined values: target area (e.g. tumors, malformations), area to be avoided (e.g. high risk functional areas, vessels), reference area (e.g. sulci, vessels, anterior and posterior commissures, basal ganglia) or surgical approach (e.g. a sulcus used as a surgical path to the target). For example, during the performance of the 
Jannin P, Raimbault M, Morandi X, Riffaud L and Gibaud B. Models of Surgical Procedures for Multimodal Image-Guided Neurosurgery, Journal of Computer Aided Surgery, 2003;8(2):98-106

step concerning the removal of the tumor, contours of a tumor may be considered as a target area, sulci segmented from T1-weighted MR imaging as reference areas, and functional data such as brain activity sources related to motor stimulations and reconstructed from a magnetoencephalographic examination as areas to be avoided.

As a starting point for the design of the model, we analyzed the descriptions made by neurosurgeons of the three types of surgical procedures listed above (i.e. SSIT, SSTC and SAH). These descriptions were either generic, i.e., referring to standardized procedures expressed as an abstraction of many concrete clinical cases, or specific, i.e., derived from the analysis of 12 clinical cases, both during and after surgery using video tapes (6 SSIT, 3 SSTC, $3 \mathrm{SAH}$ ). Results obtained with one surgeon were always discussed with at least one other, in order to fit the model with several points of view on how a specific procedure should be done. Then, we formalized this decomposition of surgical procedures into a generic model, describing the main entities and relationships. The UML formalism (Unified Modeling Language $)^{18}$ was selected among the numerous techniques available because UML is now a standard commonly used in medical information modeling (e.g. Neuronames ${ }^{4}$, UMLS ${ }^{15}$ ). Several main concepts, such as the steps and the images entities, were straightforward to formalize, whereas some others required more work and discussion. The final class diagram was iteratively obtained by assessing the model with the three types of procedures, through the translation of the clinical cases into UML object diagrams.

\section{Results}

Figure 1 presents the generic model as a UML class diagram. The major conceptual entities and relations are the following. SurgicalProcedure is the basic entity of this model. A SurgicalProcedure concerns one or more Targets and comprises one or more ordered Steps. In multimodal image-guided surgery, a procedure may require several ImageEntities corresponding to 3-D image entities (i.e. points, surfaces or volumes) extracted from 
Jannin P, Raimbault M, Morandi X, Riffaud L and Gibaud B. Models of Surgical Procedures for Multimodal Image-Guided Neurosurgery, Journal of Computer Aided Surgery, 2003;8(2):98-106

anatomical or functional preoperative images. A Target may have an ImageEntity, which is its graphical representation segmented from images. A Target has properties (e.g. size, orientation and amplitude) and is located within the right and/or left hemisphere (Side). A Target may concern several anatomical concepts (AnatInstConcept) such as a gyrus or a lobe in which it is located. A Target is also characterized by a concept denoting pathology (PathoInstConcept), such as cavernoma, glioma or epileptogenic focus. A Step comprises a single Action, which is the aim of this step (e.g. incision of the dura mater). An Action may act upon one pathological, functional or anatomical entity (InstantiatedConcept). A Step may also have an ImageEntityList, listing the anatomical and/or functional ImageEntities relevant for the performance of this specific Step. Each ImageEntity, referring to a specific ImageEntityList, has a Role representing the kind of use anticipated for that instance in that Step, such as target area, area to be avoided, reference area or surgical approach. Each ImageEntity refers to one or more InstanciatedConcepts, representing information about anatomy, function or pathology. A Step may refer to an ActionModel and may be described by one or more ActionAttributes. An ActionModel may be, for instance, a graphical element representing the action to be performed, such as contours of a craniotomy or a line representing a surgical approach to a cavernoma. An ActionAttribute provides further details on the action to be completed. For example, the action of positioning the patient may be further described by the position to be used (e.g. right or left lateral, supine or prone).

In the model, we distinguished two levels for the representation of information concerning structures involved in surgery ${ }^{23}$. The first relates to the specific patient anatomy, function or pathology (InstanciatedConcept, used as AnatInstConcept, FunctInstConcept, and PathoInstConcept) (e.g. exact anatomical location of an image entity) whereas the second refers to canonical knowledge about anatomy, pathology and function (CanonicalConcept, 
Jannin P, Raimbault M, Morandi X, Riffaud L and Gibaud B. Models of Surgical Procedures for Multimodal Image-Guided Neurosurgery, Journal of Computer Aided Surgery, 2003;8(2):98-106

used as AnatCanConcept, FunctCanConcept, and PathoCanConcept) (e.g. the precentral gyrus is an anatomical part of the frontal lobe). Instances of CanonicalConcept may eventually refer to existing knowledge about these concepts through terminology sources such as Neuronames ${ }^{4}$ included in UMLS since 1992.

\section{Validation}

\subsection{Validation Method}

A semantic validation of the model was performed by instantiating the model entities for 29 surgical procedures (Figure 2). Paper forms were designed in accordance to the generic model, containing the different classes the surgeons were asked to instantiate. In order to cope with the observers' subjectivity, these forms were filled in by four different neurosurgeons. The surgical procedures (22 SSIT, 4 SSTC and 3 SAH) were selected among clinical cases falling within the scope of the model. Of course, these clinical cases were different from those used to design the model. We designed one object diagram for each clinical case, from the corresponding paper form (Figure 3).

The surgeon could define as many steps as desired for the procedure and as many ImageEntities as ideally required to complete the action involved in each step (i.e. what kind of image information should be displayed in the oculars of a surgical microscope to perform this step ?). Surgeons were free to fill in forms with any values, but for some classes (e.g. Role, Side) we provided a list of possible values. For instance, the Role class could be instantiated with "target", "reference", "surgical approach" or "to be avoided" values. Furthermore we asked the surgeons to underline and document situations where the model could not fit the clinical requirements. 
Jannin P, Raimbault M, Morandi X, Riffaud L and Gibaud B. Models of Surgical Procedures for Multimodal Image-Guided Neurosurgery, Journal of Computer Aided Surgery, 2003;8(2):98-106

\subsection{Validation Results}

Results of the validation are given in Table 1, which includes for each surgical procedure the number of main steps, the involved anatomical, pathological and functional instantiated concepts and some comments given by operators. These results did not question the model but raised problems related to specific relation cardinalities and to the values used to instantiate the classes. The core of the model states that a surgical procedure may be broken down into a sequence of separate main steps defined by a single action and referring to a single ImageEntityList. This definition was confirmed by the surgeons. They noted that it could be of value to know when one step begins and finishes. They also suggested adding the notion of timing of each Step. They highlighted the importance of the ActionAttribute class to describe the required surgical tools or to provide more details about the type of the action (e.g. the shape of the skin incision).

The model constrained the action to act upon no more than one InstantiatedConcept. In four cases (patients 2, 4, 18 and 28 in Table 1), we realized that this cardinality had to be extended to one InstantiatedConcept or more. For instance, for a right-handed patient with a right temporal glioblastoma (patient 2), the SurgicalProcedure was a partial temporal lobectomy and the resection step concerned both the tumor and adjacent cerebral tissue. Consequently the Step acted upon two InstantiatedConcepts.

Currently, the model does not allow one to distinguish ImageEntities that are crucial to the performance of the step from optional ImageEntities. To enable this distinction, surgeons suggested adding information about the level of importance of the ImageEntity. Moreover, an accuracy value could be assigned to each ImageEntity of a Step, representing the required spatial accuracy of the ImageEntity (i.e. maximal accepted error concerning the spatial localization of the image entity, which could be compared to the error computed or estimated from the pre-processing stages). 
Jannin P, Raimbault M, Morandi X, Riffaud L and Gibaud B. Models of Surgical Procedures for Multimodal Image-Guided Neurosurgery, Journal of Computer Aided Surgery, 2003;8(2):98-106

Surgeons also noticed that one difficulty in filling the forms was related to the instance values to be used. The ActionModel class may contain a graphical representation of the action but it was sometimes difficult to find a representation different from an ImageEntity (patients 10, 14 and 21) and to differentiate the target from the action. The validation also highlighted the need for lexicons, which would contain the list of all the values that can be used to instantiate the classes. Such lexicons should be created or selected from existing ones ${ }^{5}$.

\section{Discussion}

This paper introduces a generic model of surgical procedures. It breaks down a procedure into steps and associates a goal and relevant information with each step. We restricted the scope of our model to the management of multimodal information in neuronavigation systems for craniotomies. Our primary concern was to manage multimodal information more ingeniously in order to improve and simplify multimodal image-guided craniotomies, especially when using microscope-based multimodal neuronavigation systems ${ }^{9}$. The goal was not to define a surgical procedure in exhaustive detail (e.g. taking into account surgical tools and biomechanical tissue properties), as can be done for robotic purposes. We suggest that the management of multimodal information can be improved based on prior knowledge of surgical procedures. This improvement includes two aspects: a «data management » aspect, enhancing when and why images are essential in the performance of the surgical act (e.g. place and role of images in the surgical script) and a « representation and interaction » aspect, focusing on selecting appropriate visualization and interaction features for image entities, according to their place and role in the surgical script. For instance, during surgery, only the relevant image entities belonging to the step currently being performed could be displayed, and colors selected in relation to their role. The neurosurgeon could thus better focus his or her attention to the most relevant information related to the step. 
Jannin P, Raimbault M, Morandi X, Riffaud L and Gibaud B. Models of Surgical Procedures for Multimodal Image-Guided Neurosurgery, Journal of Computer Aided Surgery, 2003;8(2):98-106

Recent works have been reported on modeling surgical procedures. Jensen et al. ${ }^{12}$ described the implementation of a microsurgical assistant. This assistant creates a surgical knowledge data base, retrieves relevant information from this data base, puts it forward to the surgeon, and keeps records about the actions or choices made by the surgeon. Munchenberg et al. ${ }^{19,20}$ introduced a system allowing the design of a surgical procedures data base in order to help robotization of maxillo-facial surgery. An instruction graph representing the surgical procedure is created (i.e. instantiated from a generic model) during the planning stage. This graph is consulted during surgery, validated after surgery and included in the data base. MacKenzie et al. ${ }^{16}$ introduced a hierarchical decomposition of laparoscopic surgical procedures and listed major interests of such a decomposition: helping the design and the evaluation of the effectiveness of new tools, allowing the training of surgeons and the evaluation of their performance, and improving planning of surgical procedures. One main limitation of this work concerns the lack of formalism or generic model supporting the hierarchical decomposition. Furthermore, none of these approaches actually models 3-D digital image information as required in the context of image-guided surgery. Surgical procedures do not only involve actions and tools, but also information about trajectories and anatomical, functional and pathological structures coming from pre-operative images and, finally, generic knowledge about these structures.

The model presented in this paper provides a framework to model information involved in the performance of neurosurgical acts and therefore leads to the definition of an ontology ${ }^{6}$ of this field of knowledge. Thanks to this model, semantics (i.e. meaning) are assigned to image data (i.e. image entities) in the context we have previously specified (multimodal image-guided craniotomies). Making this meaning explicit is a key aspect of successful information sharing between the different software components used for planning and surgery and between which 
Jannin P, Raimbault M, Morandi X, Riffaud L and Gibaud B. Models of Surgical Procedures for Multimodal Image-Guided Neurosurgery, Journal of Computer Aided Surgery, 2003;8(2):98-106

a perfect inter-operability is required. In that respect, our work could be compared to approaches dedicated to the design of medical terminologies, notably in order to facilitate codification of surgical procedures $5,7,22,26$. The main applications of these terminologies concern classifications and coding for computerized medical records, reimbursement, quality insurance, public health management, epidemiological surveys, for example.

The validation using 29 clinical cases has shown the relevance of our generic model, confirmed our main assumptions and provided us with some leads to improve the model (e.g. modifying some cardinalities, refining possible values for attributes, adding new features to the Target class, refining the distinction between instantiated knowledge and canonical knowledge). Surgeons who filled in the forms - and who had not participated in the model design - confirmed that all image entities were not necessary at every step and that the model could help managing them. Moreover, they strongly felt that the decomposition of the surgical procedure could help them to better organize it, notably during the preoperative planning stage. The study of the different clinical cases emphasized the fact that there are few variations between object diagrams based on clinical cases requiring the same kind of surgical procedure. The same steps are associated with the same actions and similar relevant image entities. Consequently, it may be worth defining models of these specific procedures, which would a priori include the expected steps and the image entities to assign. Such models would carry a priori knowledge about these procedures.

\section{Conclusions}

This paper presents a work in progress. While further validation is needed (i.e. by involving a broader set of clinical cases and surgical procedures), the initial benefits of this approach can already be outlined. It should confer real added value to the different levels of image-guided surgery, from preprocessing to planning, as well as during surgery. Models of surgical 
Jannin P, Raimbault M, Morandi X, Riffaud L and Gibaud B. Models of Surgical Procedures for Multimodal Image-Guided Neurosurgery, Journal of Computer Aided Surgery, 2003;8(2):98-106

procedures can help to better manage image data according to the surgical script, which should lead to better anticipation of surgery through the development of simulation tools. Furthermore, they may greatly improve the performance of surgery under microscope-based neuronavigation systems by optimizing both visualization and interaction features of multimodal preoperative images. Finally, such models may facilitate the generation of digital case reports and the development of teaching tools. We are convinced that our approach, in conjunction with other a priori knowledge, such as anatomical and physiological models ${ }^{25}$, will contribute to the development of decision support systems to improve information-guided therapy.

\section{Acknowledgements}

We wish to thank E. Seigneuret and Pr. J.M. Scarabin, from the Laboratoire IDM and the Neurosurgical Department, who participated in the validation of the model. This research program was supported in part by grants from the "Conseil Régional de Bretagne".

\section{References}

1. Alberstone CD, Skirboll SL, Benzel EC, et al.: Magnetic source imaging and brain surgery: presurgical and intraoperative planning in 26 patients. J Neurosurg 92 (2000) 7990

2. Apuzzo MJ: New dimensions of neurosurgery in the realm of high technology: possibilities, practicalities, realities. Neurosurgery, 38 (1996) 625-639

3. Auer LM, Radetzky PJ, Wimmer C et al.: Visualisation for planning and simulation of minimally invasive neurosurgical procedures. Medical Image Computing and Computer- 
Jannin P, Raimbault M, Morandi X, Riffaud L and Gibaud B. Models of Surgical Procedures for Multimodal Image-Guided Neurosurgery, Journal of Computer Aided Surgery, 2003;8(2):98-106

Assisted Intervention - MICCAI'99, Cambridge, UK, Chris Taylor, Alan C. F. Colchester (Eds.), Lecture Notes in Computer Science 1679 Springer (1999) 1199-1209

4. Bowden DM, Martin RF: Neuronames Brain Hierarchy. Neuroimage, 2 (1995) 63-83

5. Burgun A, Botti G, Bodenreider O, et al.: Methodology for using the UMLS as a background knowledge for the description of surgical procedures. International Journal of Biomedical Computing 43 (1996) 189-202

6. Chandrasekaran B, Josephson JR, and Benjamins VR: What are ontologies and why do we need them? IEEE Intelligent Systems January-February (1999) 20-26

7. CEN European Committee for Standardisation prEN 1828: Structure for Classification and Coding of Surgical Procedures. (1999) Brussels CEN

8. Hardenack M, Bucher N, Falk A, and Harders A: Preoperative planning and intraoperative navigation : status quo and perspectives. Comp Aid Surg 3 (1998) 153-158

9. Jannin P, Fleig OJ, Seigneuret E, et al.: A data fusion environment for multimodal and multi-informational neuronavigation. Comp Aid Surg 5 (2000) 1-10

10. Jannin P, Seigneuret E, Morandi X, et al.: Repérage sulcal et neuronavigation dans la chirurgie des cavernomes supratentoriels. Neurochirurgie 46/6 (2000) 534-540

11. Jannin P, Morandi X, Fleig OJ, et al.: Integration of sulcal and functional information for multimodal neuronavigation. J Neurosurg In Press (2002)

12. Jensen P, deJuan E, Long D, et al.: A Microsurgical Assistant for the Augmentation of Surgical Perception and Performance. Engineered System Family number 1, John Hopkins University, http://cisstweb.cs.jhu.edu/research/MicrosurgicalAssistant/

13. Kikinis R, Langham Gleason P, Moriarty TM, et al.: Computer-assisted three-dimensional planning for neurosurgical procedures. Neurosurgery 38 (1996) 640-651

14. Kockro RA, Serra L, Tseng-Tsai Y, et al.: Planning and simulation of neurosurgery in a virtual reality environment. Neurosurgery 46 (2000) 118-137 
Jannin P, Raimbault M, Morandi X, Riffaud L and Gibaud B. Models of Surgical Procedures for Multimodal Image-Guided Neurosurgery, Journal of Computer Aided Surgery, 2003;8(2):98-106

15. Lindberg DBA, Humphreys BL and McCray AT: The Unified Medical Language System. Methods of Information in Medicine 32 (1993) 281-291

16. MacKenzie CL, Ibbotson JA, Cao CGL and Lomax AJ: Hierarchical decomposition of laparoscopic surgery: a human factors approach to investigating the operating room environment. Min Invas Ther and Allied Technol 3/10 (2001) 121-127

17. Maldjian JA, Schulder M, Liu WC, et al.: Intraoperative functional MRI using a real-time neurosurgical navigation system. J Comput Assist Tomogr 21 (1997) 910-912

18. Muller PA: Modélisation objet avec UML. Eyrolles Eds (1999)

19. Munchenberg J, Worn H, Brief J, et al.: A Pattern Catalogue of Surgical Interventions for Computer-Supported Operation Planning. Medicine Meets Virtual Reality (2000), JD. Westwood, HM. Hoffman, GT. Mogel and D.Stredney Eds, IOS Press Amsterdam 227229

20. Munchenberg J, Brief J, Raczkowsky J, et al.: Operation Planning of Robot Supported Surgical Interventions. International Conference on Intelligent Robots and Systems IEEE/RSJ, Int. Conf. on Intelligent Robots and Systems (2000) ,Takamatsu, Japan 547552

21. Nimsky C, Ganslandt O, Kober H, et al.: Integration of functional magnetic resonance imaging supported by magnetoencephalography in functional neuronavigation. Neurosurgery 44 (1999) 1249-1256

22. Price C, O'Neil M, Bentley TE and Brown PJB: Exploring the ontology of surgical procedures in the Read Thesaurus. Methods of Information in Medicine 4-5/37 (1998) $420-425$

23. Rosse C, Mejino JL, Modayur BR, et al.: Motivation and Organizational Principles for Anatomical Knowledge Representation. JAMIA 5 (1998) 17-40 
Jannin P, Raimbault M, Morandi X, Riffaud L and Gibaud B. Models of Surgical Procedures for Multimodal Image-Guided Neurosurgery, Journal of Computer Aided Surgery, 2003;8(2):98-106

24. Satava RM and Carrico CJ: Advanced Simulation Technologies for Surgical Education. Bulletin of the American College of Surgeon 7/81 (1996) 71-77

25. Taylor C, Draney MT, Ku JP, et al.: Predictive medicine: computational techniques in therapeutic decision making. Comp Aid Surg 4 (1999) 231-247

26. Trombert-Paviot B, Rodrigues JM, Rogers JE, et al.: GALEN: a third generation terminology tool to support a multipurpose national coding system for surgical procedures. International Journal of Medical Informatics 58-59 (2000) 71-85 
Jannin P, Raimbault M, Morandi X, Riffaud L and Gibaud B. Models of Surgical Procedures for Multimodal Image-Guided Neurosurgery, Journal of Computer Aided Surgery, 2003;8(2):98-106

Fig. 1. UML class diagram of multimodal image-guided craniotomies

Fig. 2. Simplified UML collaboration diagram (removal of a cavernoma, patient 14)

Fig 3. Example of a paper form filled by neurosurgeons (Step 5 for patient 14) 


\begin{tabular}{|c|c|c|c|c|c|c|}
\hline $\mathbf{N}^{\circ}$ & $\begin{array}{c}\text { Surgical } \\
\text { procedure }\end{array}$ & $\begin{array}{l}\mathrm{Nb} \text { of } \\
\text { steps }\end{array}$ & Anatomical concepts & Pathological concept & Functional concepts & Comments \\
\hline 1 JaI & nnimbiBnain & ibauts M & 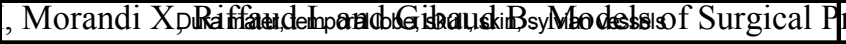 & rocedilgregsafeastiblybtibnoda & I Image-Guided Ney & Irosurgery, Journal of Computer Aided \\
\hline $2 S$ pl $_{1}$ & rgerglion $803 ;$ & $8(2) 598-$ & 06 Dura mater, temporal lobe, skull, skin, sylvian vessels, right lateral ventricle & High grade astrocytoma & - & In step of temporal lobectomy, Action acts on two Concepts. \\
\hline 3 & Metastasis & 5 & $\begin{array}{l}\text { Precentral lobe, superior sagittal sinus, central sulcus, precentral sulcus, superior frontal } \\
\text { sulcus, skin, skull, dura mater. }\end{array}$ & Metastasis & $\begin{array}{c}\text { Motor and somesthetic right } \\
\text { hand areas }\end{array}$ & - \\
\hline 4 & Glioma & 5 & Temporal lobe, lateral sulcus, skin, skull, dura mater, sylvian vessels, left lateral ventricle & Glioblastoma & Language areas & In step of anterious temporal lobectomy, Action acts on two Concepts. \\
\hline 5 & Glioma & 7 & $\begin{array}{l}\begin{array}{c}\text { Parietal lobe, skull, skin, dura mater, intraparietal sulcus, superior temporal sulcus, cortex, } \\
\text { lateral ventricle }\end{array} \\
\end{array}$ & Low grade cystic glioma & & - \\
\hline 6 & Glioma & 4 & Skin, skull, dura mater. & Gliosarcoma & - & - \\
\hline 7 & Cortectomy & 4 & Superior temporal sulcus, skin, skull, dura mater, cortex, sylvia fossa & - & - & - \\
\hline 8 & Cortectomy & 4 & $\begin{array}{c}\text { Temporal lobe, skin, skull, dura mater, petrous bone, lateral ventricle, carotid artery, } \\
\text { brainstem }\end{array}$ & - & - & - \\
\hline 9 & Cortectomy & 3 & Cortex, sylvia fossa, skull, skin, dura mater & - & - & ModelAction and an ImageEntity are the same in steps 1,2,4 and 5. \\
\hline 10 & Cavernoma & 6 & $\begin{array}{l}\text { Precentral gyrus, skin, skull, dura mater, precentral sulcus, central sulcus, frontal sulcus, } \\
\text { rolandic vessels }\end{array}$ & Cavernoma & Motor areas & - \\
\hline 11 & Cavernoma & 6 & $\begin{array}{l}\text { Superior parietal lobe, skin, skull, dura mater, post central sulcus, intraparietal sulcus, } \\
\text { rolandic veins, cortex }\end{array}$ & Cavernoma & Somesthetic areas & - \\
\hline 12 & Glioma & 5 & $\begin{array}{l}\text { Temporal lobe, skin, skull, dura mater, sylvia fossa, superior temporal sulcus, inferior horn } \\
\text { of lateral ventricle }\end{array}$ & Glioblastoma & Language areas & - \\
\hline 13 & Glioma & 5 & $\begin{array}{l}\text { Temporal lobe, occipito temporal sulcus, skin, skull, dura mater, sylvia fossa, inferior horn } \\
\text { of lateral ventricle }\end{array}$ & Glioblastoma & - & - \\
\hline 14 & Cavernoma & 5 & $\begin{array}{l}\text { Posterior temporal sulcus, inferior temporal sulcus, superior temporal sulcus, skin, skull, } \\
\text { head, dura mater, sylvia fossa. }\end{array}$ & Cavernoma & Language areas & ModelAction is used as a verbal description of the Action. \\
\hline 15 & Glioma & 5 & $\begin{array}{l}\text { Prefrontal and frontal lobes, skin, skull, dura mater, superior sagittal sinus, sylvia fossa, } \\
\text { precentral sulcus, anterior horn of lateral ventricle }\end{array}$ & Low grade oligodendroglioma & $\begin{array}{l}\text { Motor areas of right superior } \\
\text { limb }\end{array}$ & - \\
\hline 16 & Glioma & 5 & $\begin{array}{l}\text { Prefrontal lobe, skin, skull, dura mater, postcentral sulcus, coronal suture, superior sagittal } \\
\text { sinus, sylvia fossa, anterior horn of lateral ventricle, anterior cerebral artery }\end{array}$ & Glioblastoma & $\begin{array}{l}\text { Motor areas of left superior } \\
\qquad \operatorname{limb}\end{array}$ & - \\
\hline 17 & Metastasis & 5 & Frontal lobe, skin, skull, dura mater, superior sagittal sinus, precentral sulcus, sylvia fossa & Metastasis & Language and motor areas & - \\
\hline 18 & Glioma & 5 & Temporal and occipital lobes, skin, skull, dura mater, lateral sinus & Glioblastoma & - & In step of total removal, Action acts on two Concepts. \\
\hline 19 & Glioma & 5 & Temporal lobe, skin, skull, dura mater, lateral sulcus & Glioma & - & - \\
\hline 20 & Cavernoma & 6 & Superior temporal gyrus, skin, skull, dura mater, lateral sulcus, superior temporal sulcus & Cavernoma & Language and auditive areas & - \\
\hline 21 & Lobectomy & 3 & Temporal lobe, superior temporal sulcus, vein of Labbé, skin, skull & Low grade astrocytoma & Language areas & ModelAction and an ImageEntity are the same in step 2. \\
\hline 22 & Glioma & 5 & Parietal lobe, head, skin, skull, sylvia fossa, dura mater, sulcus postcentral. & Glioblastoma & $\begin{array}{l}\text { Motor, somesthetic and } \\
\text { language areas }\end{array}$ & - \\
\hline 23 & Glioma & 5 & Parietal lobe, head, skin, skull, dura mater, right ventricle & Low grade glioma & Motor and somesthetic areas & - \\
\hline 24 & Glioma & 5 & $\begin{array}{c}\text { Temporal lobe, head, sylvia fossa, skin, skull, dura mater, superior temporal sulcus, inferior } \\
\text { horn of lateral ventricle }\end{array}$ & Glioblastoma & Language areas & - \\
\hline 25 & Metastasis & 4 & Occipital lobe, head, superior sagittal sinus, lateral sinus, skin, skull. & Metastasis & Visual areas & - \\
\hline 26 & Glioma & 7 & $\begin{array}{c}\text { Frontal lobe, head, skin, skull, superior sagittal sinus, dura mater, inferior horn of lateral } \\
\text { ventricle, superior frontal sulcus, precentral sulcus }\end{array}$ & Glioblastoma & Motor and somesthetic areas & - \\
\hline 27 & Glioma & 5 & $\begin{array}{l}\text { Occipital lobe, head, skull, skin, superior sagittal sinus, dura mater, anterior horn of lateral } \\
\text { ventricle }\end{array}$ & Glioblastoma & Language areas & - \\
\hline 28 & Glioma & 5 & $\begin{array}{c}\text { Frontal lobe, head, skin, skull, superior sagittal sinus, dura mater, inferior horn of lateral } \\
\text { ventricle, superior frontal sulcus, precentral sulcus }\end{array}$ & Low grade oligodendroglioma & Motor areas & In step of total removal, Action acts on two Concepts. \\
\hline 29 & Glioma & 5 & $\begin{array}{l}\text { Internal temporal lobe, head, skin, sylvia fossa, skull, dura mater, superior temporal sulcus, } \\
\text { lateral ventricle, inferior temporal sulcus }\end{array}$ & Low grade astrocytoma & Motor Areas & - \\
\hline
\end{tabular}

Table 1. Results of validation: number of steps, anatomical, pathological and functional instantiated concepts and comments on the model 
Jannin P, Raimbault M, Morandi X, Riffaud L and Gibaud B. Models of Surgical Procedures for Multimodal Image-Guided Neurosurgery, Journal of Computer Aided Surgery, 2003;8(2):98-106 